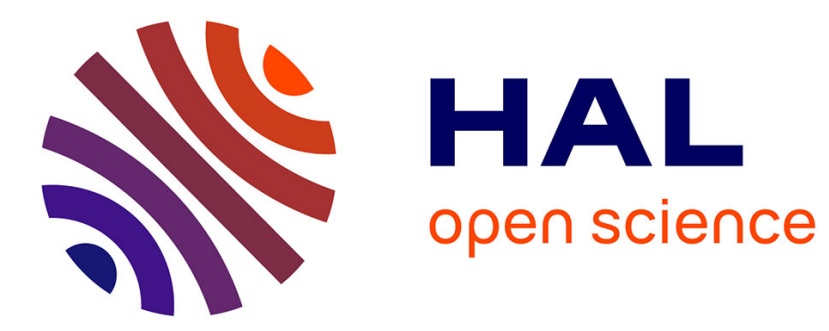

\title{
Improved photon yield from a green dye with a reducing and oxidizing system
}

\author{
Antoine Le Gall, David Dulin, Gilles Clavier, Rachel Méallet-Renault, \\ Philippe Bouyer, Karen Perronet, Nathalie Westbrook
}

\section{- To cite this version:}

Antoine Le Gall, David Dulin, Gilles Clavier, Rachel Méallet-Renault, Philippe Bouyer, et al.. Improved photon yield from a green dye with a reducing and oxidizing system. ChemPhysChem, 2011, 12 (9), pp.1657-1660. 10.1002/cphc.201100085 . hal-00597706

\section{HAL Id: hal-00597706 \\ https://hal-iogs.archives-ouvertes.fr/hal-00597706}

Submitted on 1 Jun 2011

HAL is a multi-disciplinary open access archive for the deposit and dissemination of scientific research documents, whether they are published or not. The documents may come from teaching and research institutions in France or abroad, or from public or private research centers.
L'archive ouverte pluridisciplinaire HAL, est destinée au dépôt et à la diffusion de documents scientifiques de niveau recherche, publiés ou non, émanant des établissements d'enseignement et de recherche français ou étrangers, des laboratoires publics ou privés. 


\section{Improved photon yield from a green}

\section{dye with a reducing and oxidizing}

\section{system}

\author{
Antoine Le Gall ${ }^{\$[a]}$, David Dulin ${ }^{\$[a]}$, Gilles Clavier ${ }^{[b]}$, \\ Rachel Méallet-Renault ${ }^{[b]}$, Philippe Bouyer ${ }^{[a]}$, Karen \\ Perronet $^{[\mathrm{a}]}$, Nathalie Westbrook ${ }^{\text {*a] }}$
}

Among the labels available for single-molecule fluorescence microscopy, organic dyes are still very much used thanks to their small size, well-known photophysical properties and easiness to conjugate with biomolecules. ${ }^{[1,2]}$ Were it to control their bleaching process for superresolution experiments ${ }^{[3]}$ or just to be able to monitor them for a longer time, increasing the lifetime of organic dyes has raised interest among the community. ${ }^{[2,4,5,6]}$ As oxygen is known to be one of the major factors leading to photobleaching, different strategies have been proposed to eliminate it from the studied samples. However, as oxygen is also a triplet state quencher, the result is usually an increased blinking of the fluorophore, that may be annoying for several applications such as tracking or FRET (Förster Resonance Energy Transfer). In this context, Tinnefeld and co-workers ${ }^{[2]}$ have developed a rational technique to minimize both photobleaching and blinking by removing oxygen and adding a reducing and oxidizing system (ROXS) to quench the triplet state by electron-transfer reactions. They demonstrated its high efficiency on several dye families in the yellow to red range. Extending this strategy to green dyes could be problematic since these authors also showed ${ }^{[7]}$ that an excitation at $488 \mathrm{~nm}$, more energetic than a red one, could bring a dye from the first excited singlet or triplet state to higher excited states, susceptible to lead to increased photobleaching.

Herein, we adapt this ROXS strategy to BODIPY-FI (BFI), a green dye (excitation@488nm, emission@510nm). Its very small size makes it the label of choice for biomolecular labelling when steric hindrance is concerned ${ }^{[8]}$, but its short lifetime and small emission rate have been a strong limitation so far. We show that we can increase by a factor of 20 the total number of emitted photons, as well as the lifetime of this fluorophore. Our method,

[a] A. Le Gall, Dr D. Dulin, Dr P. Bouyer, Dr K. Perronet, Prof. Dr N. Westbrook

Laboratoire Charles Fabry de I'Institut d'Optique

CNRS et Université Paris Sud 11

2 avenue Fresnel, 91127 Palaiseau cedex, France

Fax: (+33) 164533101

[b] $\operatorname{Dr}$ G. Clavier, DrR. Méallet-Renault

Laboratoire Photophysique et Photochimie Supra-Moléculaires ENS Cachan

65 avenue du président Wilson 94235 Cachan cedex, France

\$ These two authors contributed equally to this work. compatible with biological experiments, increases the interest of using BFI for single molecule experiments.

The ROXS described in [2] can be efficient on BFI, as proved by the calculations exposed in the experimental section. Its mechanism, based on electron transfer reaction between the triplet state and the redox species, is briefly depicted in Fig. 1, where ascorbic acid (AA) is the reducing agent and methylviologen (MV) the oxidizing one. The main difference with ref [2] is that we used an other oxygen scavenging system based on protocatechuic acid (PCA) combined with protocatechuate3,4 ,dioxygenase (PCD). ${ }^{[6]}$ This system is compatible with the use of cell extracts, as needed in some biological experiments, and presents no fluorescence in the green range unlike the glucose oxidase/catalase one, used in [2]. In preliminary work [9], we had observed that $\mathrm{BFI}$ could emit more photons using $\mathrm{AA}+\mathrm{MV}$ in combination with PCA/PCD. The analysis was not as detailed as what is shown here, where we analyzed not only the total lifetime before photobleaching but also the effect on blinking. The other important novelty is that we show that the use of PCA leaves the addition of AA unnecessary. Indeed, since $E_{\text {ox }}(P C A$ vs $S C E)=0.79 \mathrm{~V}^{[9]}, \mathrm{PCA}$ can also play the role of the reducing agent in ROXS. We checked experimentally that the addition of AA had no visible effect on the fluorescence properties of Bodipy $\mathrm{FI}$, and we did not use it in our study. (a)

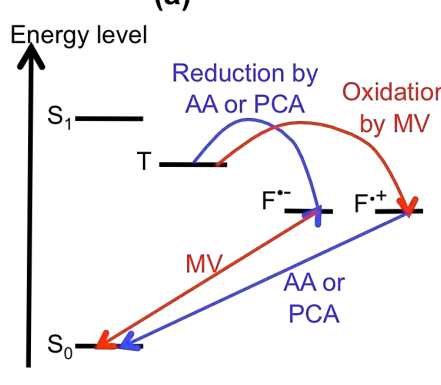

(b)

\begin{tabular}{|c|c}
\multicolumn{2}{|c|}{$(\mathbf{b})$} \\
$\begin{array}{c}\text { redox } \\
\text { couple }\end{array}$ & $\begin{array}{c}\text { redox } \\
\text { potential }(\mathrm{V})\end{array}$ \\
\hline $\mathrm{S}_{1} / \mathrm{F}^{*-}$ & 1.38 \\
$\mathrm{~F}^{*} / \mathrm{S}_{0}$ & 1.17 \\
${\mathrm{~T} / \mathrm{F}^{-}}^{-}$ & 1.10 \\
$\mathrm{AA}{ }^{++} / \mathrm{AA}$ & 0.06 \\
$\mathrm{MV} / \mathrm{MV}^{--}$ & -0.45 \\
$\mathrm{~F}$ & -1.00 \\
$\mathrm{~F} / \mathrm{T}$ & -1.07
\end{tabular}

Figure 1. (a) Action of ROXS to quench triplet state through electron transfer reactions. $S_{0}, S_{1}$ and $T$ denote resp. ground, first excited and triplet states. $\mathrm{F}^{*}$ and $\mathrm{F}^{*+}$ are the radical anion and cation. (b) Values of the redox potentials involved in the process (given vs SCE). The potentials for AA and MV come from $\operatorname{Ref}^{[10]}$ and ${ }^{[11]}$ resp. The value of the $F^{*+} / S_{0}$ oxidation potential is estimated from Ref ${ }^{[12]}$ in comparison with similar BODIPY dyes.

To compare the photophysical properties of Bodipy FI with and without the ROXS system, we performed measurements in two different environments: in a TRIS buffer (denoted hereafter buffer T) containing oxygen, and in a buffer $T$ depleted in oxygen with PCA/PCD and where an oxidizing agent MV was added (called buffer R).

In buffer T, BFI fluorescence exhibits a poor signal to noise ratio (SNR) compared to cyanine dyes such as Cy3 or Cy5. At moderate excitation intensities $\left(\sim 700 \mathrm{~W} / \mathrm{cm}^{2}\right)$ it is however possible to observe single BFI with SNR $\sim 5$. When we deplete buffer $T$ of its oxygen, it has a dramatic effect on BFI fluorescence, making it almost undetectable. Flushing the sample with buffer $T$ restores the fluorescence signal. On the other hand, using buffer $\mathrm{R}$ increases $\mathrm{BFI}$ fluorescence lifetime up to second timescales, leading to a larger number of photons emitted by a 
single BFI before it bleaches. This confirms that merely eliminating oxygen reduces photobleaching but brings BFI into a metastable dark state. With the addition of the oxidizing agent MV which, combined to the reducing agent PCA, replaces oxygen as a triplet state quencher, we get both a longer lifetime and more photons out of our green fluorophore.

In order to quantify the improvement in the fluorescence properties of BFI using buffer $\mathrm{R}$, we recorded the time evolution of many single BFI fluorescence intensities using our objectivebased TIRFM set-up. Each wide-field image contained 200 to 400 molecules exhibiting a SNR higher than 4, that we observed over a duration long enough so that they would all be photobleached by the end of the film. Using 2 or 3 image series, we analyzed 590 molecules in buffer $T$ and 800 in buffer R. In Table 1, we report their fluorescence properties for a power of $20 \mathrm{~mW}$ at the entrance of the microscope, i.e. an excitation intensity at the center of the Gaussian beam of $450 \mathrm{~W} / \mathrm{cm} 2$. Note that because of the Gaussian-shaped excitation beam, molecules are excited at different intensities, depending on their location in the excitation area. In the following, we will discuss the different characteristics presented in table 1.

\begin{tabular}{|lcc|}
\hline \multicolumn{3}{|l}{ Table 1. Characteristics of fluorescence emission of BFI in buffers T and R } \\
& Buffer T & Buffer R \\
\hline $\begin{array}{l}\text { Total number of emitted } \\
\text { photons }\end{array}$ & $5000 \pm 500$ & $100000 \pm 7000$ \\
\hline Total lifetime (s) & $0.20 \pm 0.02$ & $3.7 \pm 0.3$ \\
\hline $\begin{array}{l}\text { Emitted photons during first } \\
\text { ON time }\end{array}$ & & $47000 \pm 3000$ \\
\hline First ON time (s) & & $1.8 \pm 0.1$ \\
\hline
\end{tabular}

Figure 2 shows the probability for $\mathrm{BFI}$ to emit $\mathrm{N}$ photons before photobleaching in buffers $T$ and $R$. From an exponential fit of each plot, we can deduce the mean total number of emitted photons: 5000 in buffer $T$ and $10^{5}$ in buffer R. This represents a gain of 20 .

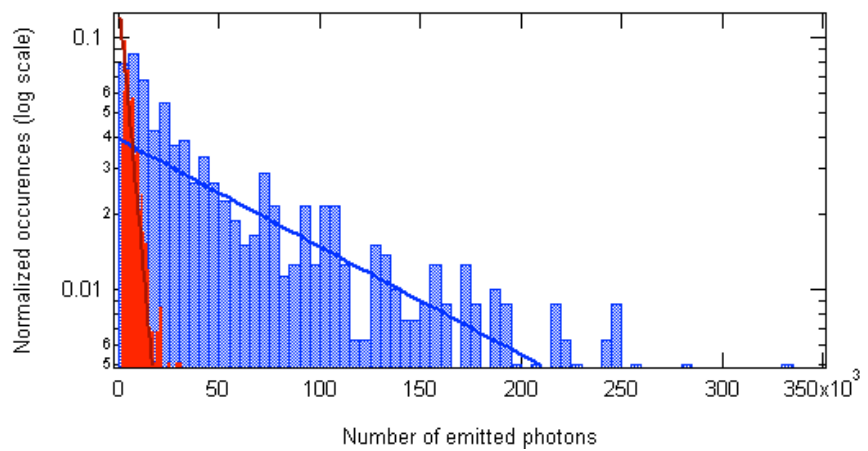

Figure 2. Histograms showing the probability for a BFI to emit $\mathrm{N}$ photons in buffer $\mathrm{T}$ (red bins) and R (blue bins).

We analyzed in the same way the lifetime of the fluorophores before photobleaching. In buffer $\mathrm{T}$, bleaching occurs typically during the first or second recorded image (one image $=100 \mathrm{~ms}$ ) and almost no blinking can be observed with our temporal resolution. The longer lifetime in buffer $\mathrm{R}$ is sometimes accompanied by the appearance of blinking events, such as the one shown in Fig 3. The total lifetime reported in table 1 takes into account the sum of the ON times. The mean lifetime is $0,20 \mathrm{~s}$ in buffer $\mathrm{T}$ and increases to $3,7 \mathrm{~s}$ in buffer $\mathrm{R}$, almost a factor of 20 larger. Among the 800 molecules studied in buffer R, $57 \%$ blinked. However, even if we compare the emission during the first ON time in buffer $\mathrm{R}$ to the total emission in buffer $\mathrm{T}$, there is already a gain by a factor of 9 in total number of photons $(47000$ vs 5000$)$ and in duration $(1,8 \mathrm{~s}$ vs $0,2 \mathrm{~s})$. We calculated the arithmetic mean over all the molecules that blinked at least once, and found a mean number of blinking events of 5 and a mean duration for ON and OFF times (apart from the first ON) of 0,6s.

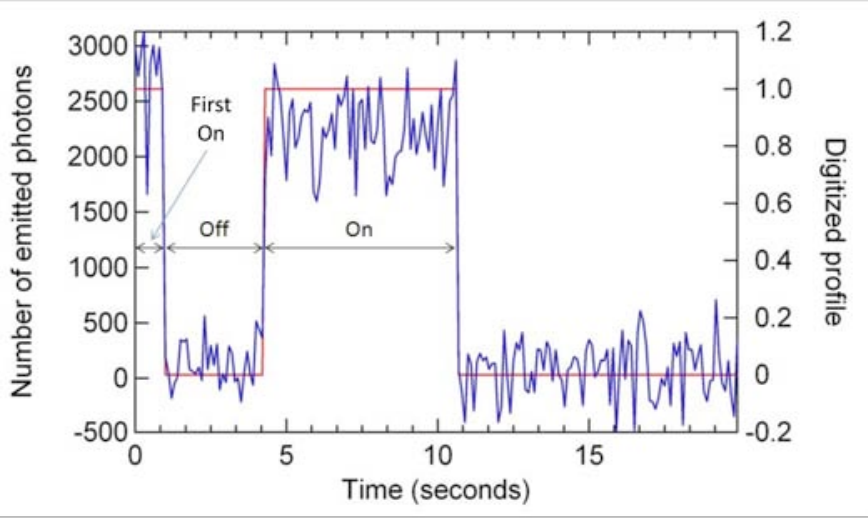

Figure 3. Example of an intensity versus time trace of BFI in buffer R (blue) and its digitalized trace (red), defining ON and OFF times. This fluorophore blinked once, i.e. there was one OFF and one ON times after the first ON.

Taking advantage of our TIRFM method, we compared the saturation in the emission of single BFI in buffers $T$ and $R$. Figure 4 shows the fluorescence rate $R_{\text {fluo }}$ during the first $O N$ time versus the excitation intensity $l_{\text {exc }}$. The fluorescence rate is calculated from the number of photons emitted in a $100 \mathrm{~ms}$ image divided by the duration of that image. The local excitation intensity for each molecule is deduced from a gaussian fit of the coverslip fluorescence, which is proportional to the excitation intensity (see details in the exp. section). In the displayed data we observe a high dispersion that can be ascribed partly to blinking events shorter than the duration of one image but mainly to non uniformities of the evanescent wave that are not taken into account in the Gaussian fit of the coverslip fluorescence. Those non uniformities are due to scattering and diffraction of the coherent laser light at every optical component on the optical path leading to interference fringes. ${ }^{[13]}$

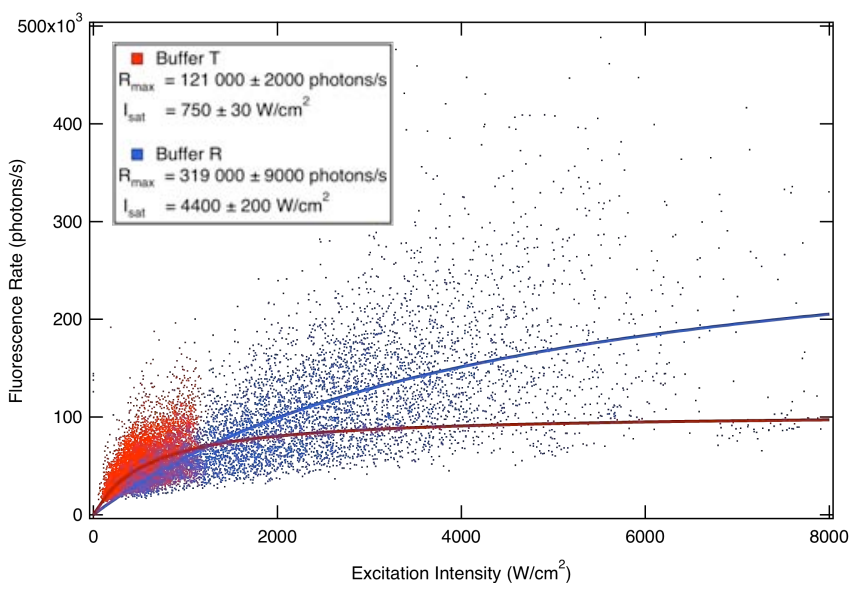

Figure 4. Fluorescence rate vs excitation intensity in buffer $\mathrm{T}$ (red) and $\mathrm{R}$ (blue). Dots show experimental values and plain curves the fitting functions.

Experimental data are well fitted by a saturation curve of the form $R_{\text {fluo }}=R_{\max } /\left(1+I_{\text {sat }} / l_{\text {exc }}\right), \quad R_{\max }$ and $I_{\text {sat }}$ being two adjustable parameters. ${ }^{[14]}$ The initial slope of the saturation curve in buffer $T$ 
is steeper, meaning that for low excitation intensities the fluorescence rate is higher in buffer $\mathrm{T}$ than in buffer $\mathrm{R}$ during the first $100 \mathrm{~ms}$ of excitation. However, saturation occurs quickly in buffer $\mathrm{T}$, probably due to photobleaching, so that above $1,35 \mathrm{~kW} / \mathrm{cm} 2$, the fluorescence rate becomes higher in buffer $R$. Of course, this higher fluorescence rate will correspond to a shorter lifetime before photobleaching than reported in table 1 . In fine, whether we want a long lifetime to observe our fluorophores over a long period, or a high fluorescence rate for precise localization, the use of buffer $\mathrm{R}$ clearly improves the conditions of observation of single Bodipy FI molecules.

To conclude, in this communication, we have shown that ROXS, proposed and tested by Tinnefeld on yellow to red emitting dyes, applies also to a green label, BODIPY-FI. Furthermore, in this case, the addition of the reducing agent is unnecessary thanks to the use of PCA/PCD as an oxygen scavenger. In such an environment, BFI acquires a photostability and a brightness fulfilling the criteria for single molecule observation on the time scales of many biological processes.

Our strategy could be applied to other dyes which have a similar photobleaching path, providing the redox potentials of the species and the $E_{0,0}$ energy are known. The main limitation would come from an instable radical anion or cation that would react too rapidly with the buffer through a different path. In the Bodipy family, the redox potentials vary by only $-1 \mathrm{~V}$ for reduction and +1V for oxidation as shown in [17], so that PCA could act as a reductant for Bodipy up to an emission wavelength of ?? Above, the ROXS strategy could still work but with the addition of AA. We have also tested experimentally our ROXS on another green dye, the Alexa 488, for which the redox potentials are not known. This fluorophore has a longer lifetime in TRIS than Bodipy FL, but this lifetime can still be

\section{Experimental Section}

Specific binding of BODIPY-FL to a microscope coverslip is done through labelled olinucleotides attached via a biotin-streptavidin linkage. Sample preparation has been described elsewhere [9].

The two buffers used to study BODIPY-FL are buffer $T$ alone, and buffer $T$ depleted in oxygen plus an oxidizing agent $(R)$. The oxygen scavenger used for oxygen depletion is protocatechuic acid (PCA, Sigma) combined with protocatechuate-3,4, dioxygenase (PCD, Sigma) (PCA 10mM / PCD 200nM), prepared as described in [6]. We use 1,1'-dimethyl-4,4'-bipyridinium dichloride hydrate $(\mathrm{MV}, 0.5 \mathrm{mM}$, Sigma) as an oxidizing agent, as suggested in [2]. By comparing the redox potentials of each species, we first checked if the ROXS described in [2] is efficient on $\mathrm{BFI}$, using the same notations (see Fig 1). The BFI zero-zero energy $E_{0,0}$ is $2,45 \mathrm{eV}$ for $I_{\max }(a b s) \approx 502 \mathrm{~nm}$. We assume that triplet state energy is $0.28 \mathrm{eV}$ less (as for $\mathrm{Cy} 5^{[2]}$ ). In the presence of a reducing agent, $\mathrm{AA}$ and an oxidizing one, $\mathrm{MV}$, two processes may quench $T$ state and thus reduce its lifetime. $A A$ can first reduce $T$ state in $\mathrm{F}^{-}$. This reaction is thermodynamically possible since the free energy is $\Delta G=e\left(E_{o x}-E_{r e d}\right)-E_{0,0}+C=-1.04 \mathrm{eV}$ (calculated with Rehm-Weller equation ${ }^{[15]}$, assuming that $\mathrm{C}$, which is the solventdependent Coulombic attraction energy, is here negligible because water is a solvent with a high polarity). Then, MV reacts with $F^{*-}(\Delta G=$ $0.62 \mathrm{eV}$ ) to bring $\mathrm{BFI}$ back in its ground state. MV may also first oxidize $T$ state in $F^{*+}(\Delta G=-0.55 \mathrm{eV})$. Then, $A A$ reacts with $F^{*+}(\Delta G=-$ $1.11 \mathrm{eV}$ ) to bring BFI back in its ground state. In our case, AA is replaced by $\mathrm{PCA}$ itself.

Experiments are carried out using an inverted microscope (Olympus IX70) set up for total internal reflection fluorescence microscopy (TIRFM). The sample is excited by a $488 \mathrm{~nm}$, TE linearly polarized, $\mathrm{CW}$ laser beam (488-200 CRDH, Coherent) expanded and focused off-axis in the back focal plane of an oil-immersion objective (Olympus ApoPlan $x 60, N A=1.45$ ). The evanescent wave generated from the total reflection of the laser beam covers an elliptical area of diameters $100 \mu \mathrm{m}$ and $80 \mu \mathrm{m}$ on the interface. The fluorescence signal collected from the excited molecules is separated from the excitation laser with a dichroic mirror and a band-pass filter (Q505lp and HQ535/50, respectively, Chroma Technology Corp.) and recorded on an EMCCD camera (Hamamatsu C9100). Acquisition is synchronised with sample illumination to prevent premature photobleaching of the molecules. Image series of $100 \mathrm{~ms}$ each separated by $100 \mathrm{~ms}$ of nonillumination of the sample were acquired.

Stacks of 200 images giving the BODIPY-FI fluorescence signal with time are analysed by a home-made Igor Pro program. Single fluorophores are identified from their diffraction-limited image size and their photobleaching in one step. Their time traces are digitized into fluorescent and non-fluorescent states for blinking analysis. From these traces we distinguish the first $\mathrm{ON}$ state, and the subsequent OFF and ON states, as shown in fig.3. Background, due to the glass coverslip fluorescence, is averaged over the last 10 pictures of the stacks (after all BFI have been bleached) and then subtracted from the whole series of images before data analysis. This background is proportional to the incident laser beam intensity, so we can use it to determine the local excitation intensity of each dye. Since the laser beam is Gaussian, the sample area displays various excitation intensities and it is thus possible to plot the BODIPY-FI fluorescence rate versus its excitation intensity calculated from the local averaged fluorescence background. All excitation intensities given in this paper take into account the Fresnel transmission coefficient at the interface ( 1,8 for our incidence angle of $70^{\circ}$ and our TE polarization).

\section{Acknowledgements}

This work has been supported by the Region Ile-de-France in the framework of C'Nano IdF. C'Nano IdF is the nanoscience competence center of Paris Region, supported by CNRS, CEA, MESR and Region lle-de-France.

Keywords: biophysics; electron transfer; fluorescence; photophysics; single molecule studies

[1] U. Resch-Genger, M. Grabolle, S. Cavaliere-Jaricot, R. Nitschke, T. Nann, Nat. Meth. 2008, 5, 763

[2] J. Vogelsang, R. Kasper, C. Steinhauer, B. Person, M. Heilemann, M. Sauer, P. Tinnefeld, Angew. Chem. Int. Ed. 2008, 47, 5465.

[3] J. Vogelsang, C. Steinhauer, C. Forthmann, I. H. Stein, B. PersonSkegro, T. Cordes, P. Tinnefeld, ChemPhysChem, 2010, 11, 2475.

[4] I. Rasnik, S. A. McKinney, T. Ha, Nat. Methods 2006, 3, 891

[5] C. E. Aitken, R. A. Marshall, J. D. Puglisi, Biophys. J. 2008, 94, 1826.

[6] R. Dave, D. S. Terry, J. B. Munro, S. C. Blanchard, Biophys. J. 2009, 96, 2371.

[7] R. Kasper, M. Heilemann, P. Tinnefeld, M. Sauer, Proc. SPIE 2007, 6633, 663312

[8] K. Perronet, P. Bouyer, N. Westbrook, N. Soler, D. Fourmy, S. Yoshizawa, J. Lumin. 2007, 127, 264 and references therein.

[9] D. Dulin, A. Le Gall, K. Perronet, N. Soler, D. Fourmy, S. Yoshizawa, P. Bouyer, N. Westbrook, Physics Procedia 2010, 3, 1563.

[9] M. A. Miranda, F. Galindo, A. M. Amat, A. Arques, Applied Catalysis B: Environmental, 2001, 30, 437.

[10] C. R. Lambert, I. E. Kochevar, Photochem. Photobiol. 1997, 66, 15

[11] G.J. Kavarnos, Fundamentals of Photoinduced Electron Transfer, Wiley, New York (1993).

[12] R. Y. Lai, A. J. Bard, J. Phys. Chem. B 2003, 107, 5036.

[13] R. Fiolka, Y. Belyaev, H. Ewers, A. Stemmer, Microscopy Research and Technique, 2008, 71, 45.

[14] Note that $R_{\text {fluo }}$ is underestimated when a fluorophore has bleached before the end of the first image, which will occur at high enough 
excitation intensities. $R_{\max }$ is thus most likely underestimated and $I_{\text {sat }}$, i.e.

the excitation intensity necessary to reach $R_{\max } / 2$, is also lower than the real saturation intensity.

[15] D. Rehm, A. Weller, Isr. J. Chem. 1970, 8, 259. 